\title{
Disturbance Rejection Ability Enhancement Using Repetitive Observer in Phase-locked Loop for More Electric Aircraft
}

\author{
Mi Tang \\ Power Electronics, Machine and \\ Control (PEMC) group \\ University of Nottingham \\ Nottingham, UK \\ mi.tang2@nottingham.ac.uk \\ Andrea Formentini \\ Dept. of Electrical and Electronic \\ Engineering \\ University of Nottingham \\ Nottingham, UK \\ andrea.formentini@nottingham.ac.uk
}

\author{
Stefano Bifaretti \\ Dept. of Industrial Engineering \\ University of Rome Tor Vergata \\ Rome, Italy \\ stefano.bifaretti@uniroma2.it \\ Shafiq Odhano \\ School of Engineering \\ University of Newcastle \\ Newcastle, UK \\ shafiq.odhano@newcastle.ac.uk
}

\author{
Sabino Pipolo \\ Power Electronics, Machine and \\ Control (PEMC) group \\ University of Nottingham \\ Nottingham, UK \\ sabino.pipolo@nottingham.ac.uk \\ Pericle Zanchetta \\ Dept. of Electrical and Electronic \\ Engineering \& Dept. of Electrical \\ University of Nottingham \& University \\ of Pavia \\ Nottingham, UK \& Pavia, Italy \\ pericle.zanchetta@nottingham.ac.uk
}

\begin{abstract}
Under the concept of transportation electrification, more electric aircraft (MEA) involves more electrical energy to reduce emissions. Phase-looked loops (PLLs) have been well developed for synchronizing different power sources in a grid. Since MEA operates at variable frequency from $360 \mathrm{~Hz}$ to $800 \mathrm{~Hz}$, a third-order model based steady-state linear Kalman filter PLL (SSLKF-PLL) has been proposed in literature to achieve fast tracking performance during such grid frequency variations. To suppress the potential disturbances due to harmonics in the grid, sensor scaling errors/unbalances and d.c offsets while maintaining low computational burden, this paper aims to enhance the disturbance rejection ability of SSLKF-PLL by adding a repetitive observer (RO). Simulation tests show that RO allows stable and effective suppression of disturbances from all above-mentioned sources during variable frequency operation.
\end{abstract}

Keywords - repetitive control, phase-locked loops, more electric aircraft, power system harmonics, fault tolerant control

\section{INTRODUCTION}

The move towards more electric aircraft (MEA) under the concept of transportation electrification requires the use of more electrical power to partly or completely replace the mechanical, hydraulic and pneumatic power. Taking the military standards MIL-STD-704 for example, one of the main power buses should be a 115 Vac three-phase bus with its frequency varying from $360 \mathrm{~Hz}$ to $800 \mathrm{~Hz}$. Accurate and fast tracking of phase and frequency of the three-phase voltages generated from multiple power sources is important for grid synchronization.

A distinctive three-phase PLL is desired to achieve quick response to phase jumps with strong rejection ability against disturbance due to harmonics in grid, sensor scaling errors/unbalances, d.c. offsets in the grid. Besides, for MEA, the PLL also need to be adaptive to variable frequency.

Vast majority of the existing PLLs in literature are proposed for constant-frequency grid with slight grid frequency variations allowed. If the operation frequency is too far from the design point, the performance of those constantfrequency PLLs will degrade. For MEA, according to the recent research in [1], the key shortcomings for the few available variable frequency PLLs techniques can be summarized as the follows:

- $\quad$ Adaptive observer [2] and sliding mode observer [3] based method cannot handle the d.c. offsets.

- Discrete Fourier transform (DFT) [4] based method has heavy computational burden.

- Complex least mean square [5] based method cannot maintain its performance with grid unbalances.

Therefore, authors in [1] has proposed an estimator to solve the d.c offsets issue. However, the harmonics in grid has not been considered in [1].

A third-order model based PLL, namely the steady state linear Kalman filter PLL (SSLKF-PLL), has been proposed in [6-9]. Fast tracking performance during grid frequency variation is achieved for MEA in [9] by the SSLKF-PLL because the Kalman filter not only observes the grid frequency, but also observes and compensates the acceleration of the grid frequency. It is also confirmed in [9] that SSLKFPLL has faster dynamic than the DFT based method in [4]. Regarding the disturbance rejection ability, although SSLKFPLL can reject the disturbance by reducing its control bandwidth, it cannot remove the disturbance completely. Therefore, this motivates the use of a repetitive observer (RO).

RO is first proposed in [10] for motor drive applications. Benefiting from its disturbance-observer-like structure, it can be designed independently from the feedback controller of the system. Another big convenience of using RO is that no preknowledge of the target disturbance is required. It will selflearn and tackle disturbances at all harmonics within the Nyquist frequency. What is more, with the development of high-performance control platform [11], the extra execution time required for RO would be about 5 to $10 \mu \mathrm{s}$. Thus, RO is can be a promising add-on tool for the intended PLL.

Hence, this paper proposes a novel dual-observer PLL (DO-PLL). In section II, the SSLKF-PLL will be reviewed. In section III, the proposed DO-PLL will be discussed. The case study section in IV will discuss how the parameters can be selected. Simulation tests in V will show that the proposed DO-PLL can achieve superior grid frequency and phase 


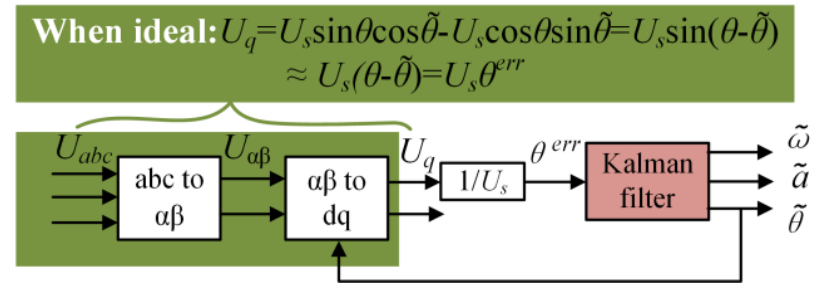

(a) SSLKF-PLL

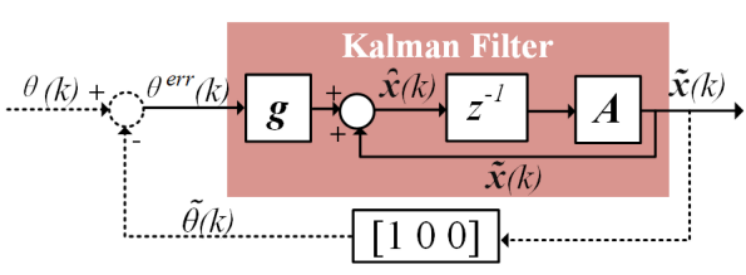

(b) Small-signal model of (a)

Fig.1: Diagrams of the SSLKF-PLL.

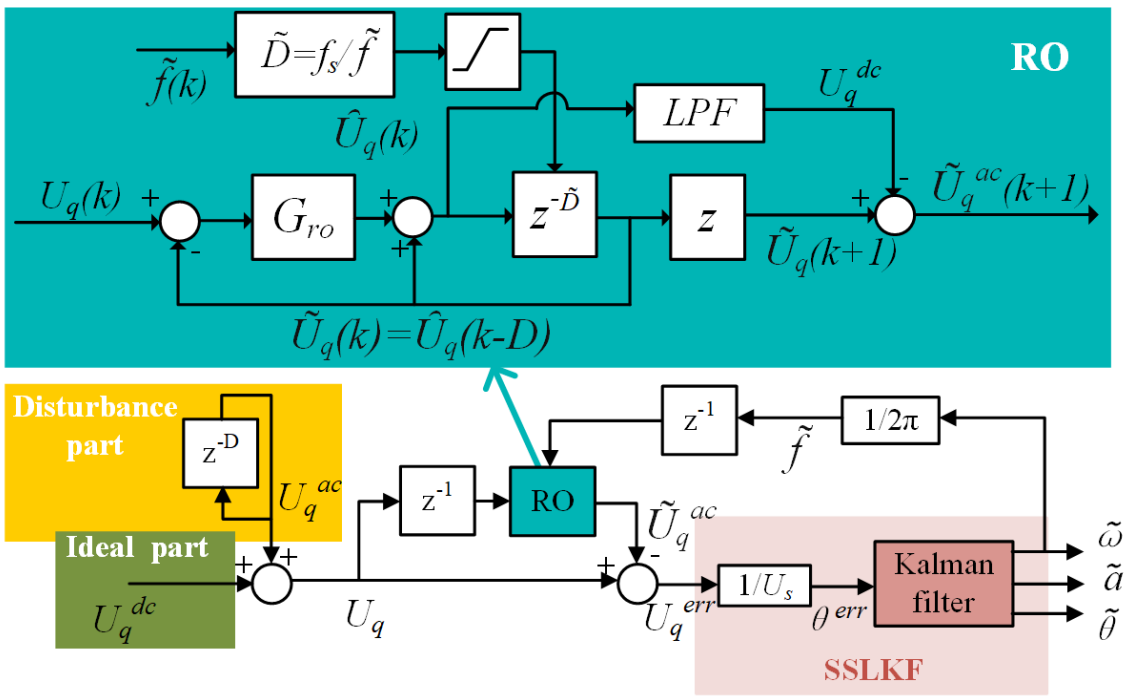

Fig.2: Small-signal model of the proposed dual-observer PLL.

tracking performance in presence of harmonics in the threephase voltages, sensor scaling errors/unbalances, sensor offset errors, while maintaining the fast response of the Kalman filter during phase jumps and grid frequency variations. The paper will be concluded in section VI.

\section{REVIEW OF SSLKF-PLL}

The block diagram and small-signal model of SSLKFPLL are drawn in Fig.1 a and Fig.1b, respectively. Where, $U_{a}$, $U_{b}, U_{c}$ are the measured three-phase voltages. $U_{\alpha}, U_{\beta}$ are the alpha-beta-axis voltages. $U_{d}$ and $U_{q}$ are the dq-axis voltages. $U_{s}$ is the peak value of the phase-to-neutral voltage. Define the phase $\theta$, grid frequency $\omega$ and grid frequency acceleration $a$ as the three state variables in vector $\boldsymbol{x}, \boldsymbol{x}=\left[\begin{array}{ll}\theta & \omega\end{array}\right]^{\mathrm{T}}$, the Kalman filter observes the three variables as $\tilde{\boldsymbol{x}}=\left[\begin{array}{lll}\tilde{\theta} & \tilde{\omega} & \tilde{a}\end{array}\right]^{\mathrm{T}}$. Matrix $\boldsymbol{A}$ is derived based on the third-order model as in (1). Vector $\boldsymbol{g}=\left[g_{l}\right.$ $\left.\begin{array}{ll}g_{2} & g_{3}\end{array}\right]^{\mathrm{T}}$ is the observer gain vector which can be tuned according to the procedure in [12] by specifying the desired poles.

$$
\boldsymbol{A}=\left[\begin{array}{ccc}
1 & T_{s} & T_{s}^{2} / 2 \\
0 & 1 & T_{s} \\
0 & 0 & 1
\end{array}\right]
$$

The closed loop transfer functions of the $\tilde{\theta}, \tilde{\omega}, \tilde{a}$ can be derived from Fig. 1b as in (2-4).

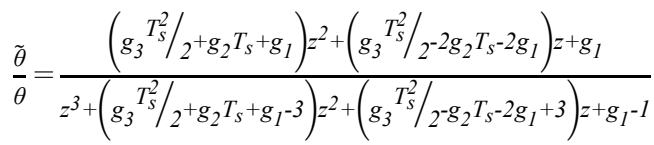

$$
\begin{aligned}
& \frac{\tilde{\omega}}{\theta}=\frac{\left(g_{3} T_{s}+g_{2}\right) z^{2}+\left(-2 g_{3} T_{s}-2 g_{2}\right) z+g_{2}}{z^{3}+\left(g_{3} T_{s}^{2} / 2^{+} g_{2} T_{s}+g_{1}-3\right) z^{2}+\left(g_{3} T_{s}^{2} / 2^{-} g_{2} T_{s}-2 g_{1}+3\right) z+g_{I^{-1}}}
\end{aligned}
$$

$$
\frac{\tilde{a}}{\theta}=\frac{g_{3^{2}} z^{2}-2 g_{3} z^{+} g_{3}}{z^{3}+\left(g_{3} T_{s}^{2} / 2^{+} g_{2} T_{s}+g_{I^{-}}\right) z^{2}+\left(g_{3} T_{s}^{2} / 2^{-} g_{2} T_{s}-2 g_{I}+3\right) z+g_{I^{-1}}}
$$

According to [12], for a given natural frequency $\omega_{n}$, the three poles in (2-4) can be placed in s domain as one real pole and two complex poles at $s_{0}=-\omega_{n}, s_{1,2}=\left(-\omega_{n} \zeta \pm j \omega_{d}\right)$, respectively, where, $\omega_{d}=\sqrt{1-\zeta^{2}} \cdot \zeta=0.9659$ is chosen in this paper.

The poles can be mapped at $\rho_{0}$ and $\rho_{1} e^{ \pm j \phi}$ in $\mathrm{z}$ domain, where, $\rho_{0}=e^{-\omega_{n} T_{s}}, \rho_{1}=e^{-\omega_{n} \zeta T_{s}}, \phi=\omega_{d} T_{s}$. Gains $g_{1}, g_{2}, g_{3}$ can be calculated as in (5).

$$
\left\{\begin{array}{c}
g_{3}=\frac{c_{1}+c_{0}+c_{2}+1}{T_{s}^{2}} \\
g_{2}=\frac{c_{1}-c_{0}+3 c_{2}+5}{2 T_{s}}-g_{3} T_{s} \\
g_{1}=c_{2}+3-g_{2} T_{s}-\frac{g_{3} T_{s}^{2}}{2}
\end{array},\left\{\begin{array}{c}
c_{0}=-\rho_{0} \rho_{1}^{2} \\
c_{1}=2 \rho_{0} \rho_{1} \zeta+\rho_{1}^{2} \\
c_{2}=-\rho_{0}-2 \rho_{1} \zeta
\end{array}\right.\right.
$$

It is worth pointing out that the dash parts in Fig. $1 \mathrm{~b}$ is based on the approximation in Fig.1a that with perfectly sinusoidal three-phase voltages and when phase error $\theta^{\text {err }}$ is small, $U_{q} / U_{s} \approx \theta-\tilde{\theta}$. However, when there is disturbance, $U_{q}$ will contain extra harmonics. Therefore, this approximation may not be true. The equivalent diagram considering disturbance will be discussed in the following section.

\section{THE PROPOSED DUAL-OBSERVER PLL}

As analyzed in [13], $U_{q}$ will contain a variety of harmonics if the three-phase voltages are non-ideal. In summary: 
- Odd harmonics in the three-phase grid leads to even harmonics in $U_{q}$.

- Scaling error of current sensors or unbalances in the grid produces $2^{\text {nd }}$ harmonic in $U_{q}$.

- Offsets in the three-phase voltages cause $1^{\text {st }}$ harmonic in $U_{q}$.

It is also concluded in [13] that the phase can still be tracked if the PLL can ignore the harmonics and force just the d.c. part of $U_{q}$ to zero.

Therefore, in Fig.2, the state $U_{q}$ is modeled by two parts: the ideal part and the disturbance part. The ideal part can be approximated as the d.c. part, while the disturbance part $U_{q}^{a c}$ includes multiple orders of harmonics, of which the fundamental period is defined as $D T_{s} . D T_{s}$ equals the grid frequency $f$, where, $T_{s}=1 / f_{s}$, is the sampling period. $f_{s}$ is the sampling frequency. $D$ can be a fractional number.

Hence, in the proposed dual-observer PLL, the RO is designed to cancel only the a.c. part of the q-axis voltage $U_{q}$. The remaining part of $U_{q}^{\text {err }}$ will be forced to zero by the same Kalman filter as in Fig.1b. The transfer function of RO is as in (6).

$$
R O(z)=\frac{\widetilde{U}_{q}^{a c}}{U_{q}}=\frac{G_{r o}\left[z^{-\widetilde{D}+1}-L P F(z)\right]}{1-\left(1-G_{r o}\right) z^{-\widetilde{D}}}, \quad \widetilde{D}=f_{s} / \tilde{f}
$$

Where, the length of the delay chain $z^{-\tilde{D}}$ is updated in realtime by the observed grid frequency $\tilde{f}$. Since $\tilde{D}$ can be a fractional number. $G_{r o}$ is the gain of RO. The $L P F(z)$ is a lowpass filter to calculate the d.c. part in $U_{q}$. A sixth order Lagrange fractional delay filter as in [13] has been adopted in this paper to implement the fractional delay chain as in (7).

$$
\begin{gathered}
\widehat{U}_{q}(k-\widetilde{D})=\sum_{i=0}^{6} P_{i} \widehat{U}_{q}\left(k-\widetilde{D}_{0}-i\right) \\
P_{i}=\prod_{\substack{h=0 \\
h \neq i}}^{6} \frac{\widetilde{D}_{f}-h}{i-h}, \text { for } i=0,1, \ldots, 6
\end{gathered}
$$

Where, $\tilde{D}_{0}$ and $\tilde{D}_{f}$ denote the integer part and fractional part of $\tilde{D}$, respectively. $\hat{U}_{q}(k-\tilde{D})$ is the estimated $U_{q}$ at $t_{k-\tilde{D}}$.

It can be seen from Fig. 2 that adding RO does not affect the Kalman filter part of the original SSLKF-PLL. Thus, the small-signal model in Fig. 1b still applies in the proposed DOPLL, and the transfer function of the Kalman filter part remains the same as in (2-4). The $\left(1-R O(z) z^{-1}\right)$ part in Fig.2 can be understood as another filter to remove any harmonics with Nyquist frequency. The bode plot of this part will be provided in the next section.

Overall, there are four parameters to be designed in the proposed DO-PLL: the sampling frequency $f_{s}$, the RO observer gain $G_{r o}$, the lowpass filter $\operatorname{LPF}(z)$, the bandwidth of the Kalman filter loop. The design procedure for these four parameters are discussed in the following section.

\section{CAse Studay}

Considering the worst condition when the three-phase grid suffers from harmonic distortions, sensor scaling error/unbalances, and d.c. offsets at the same time, the following imperfections are contained in the three-phase voltages for testing:
1) $3 \% 3^{\text {rd }}$ harmonic, $4 \% 5^{\text {th }}$ harmonic, $3 \% 7^{\text {th }}$ harmonic, $1.5 \% 9^{\text {th }}$ harmonic, $3.5 \% 11^{\text {th }}$ harmonic and $3 \% 13^{\text {th }}$ harmonic in $U_{a b c}$.

2) Phase $b$ has an offset of 5\%.

3) The amplitude of phase $c$ is 5\% smaller than the other two phases.

The resultant unified three-phase voltages at $360 \mathrm{~Hz}$ are shown in Fig.3.

\section{A. The choice of $f_{s}$}

Since the $13^{\text {th }}$ harmonics of the maximum $800 \mathrm{~Hz}$ grid is $10.4 \mathrm{kHz}, 20.8 \mathrm{kHz}$ sampling frequency is the minimum requirement according to principles of sampling. Therefore, $f_{s}=80 \mathrm{kHz}$ is chosen, such that there are 7.7 samples per period for the $13^{\text {th }}$ harmonics.

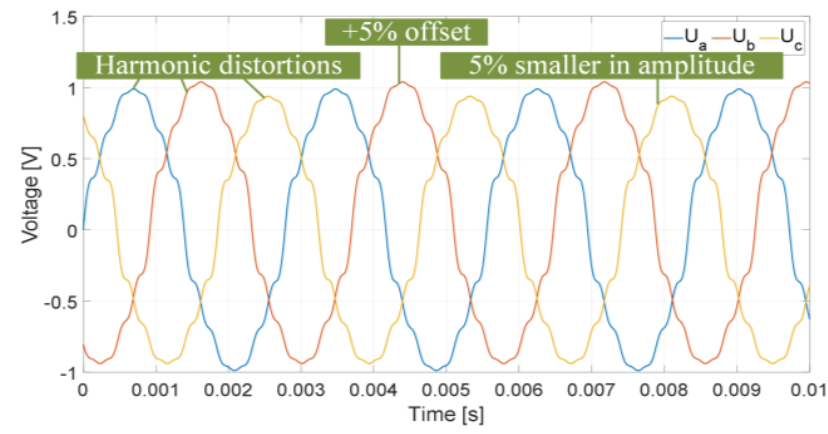

Fig.3: The unified three-phase voltages at $360 \mathrm{~Hz}$.

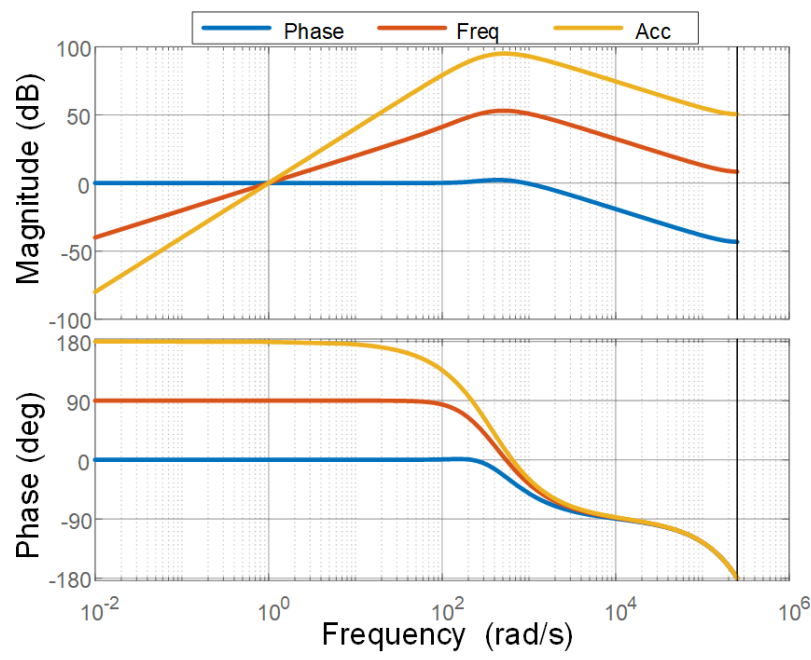

Fig.4: Bode plots for the observed phase, angular frequency and acceleration without RO.

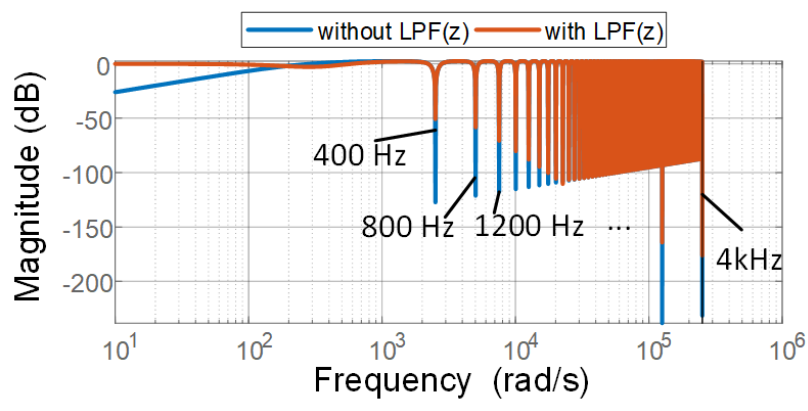

Fig.5: Amplitude response of $(1-R O(z) / z)$ with grid frequency is $400 \mathrm{~Hz}$ and $D=200$ with and without $L F P(z)$. 


\section{B. The choice of the Kalman filter bandwidth}

The natural frequency of the Kalman filter is kept the same as in [9], i.e. $60 \mathrm{~Hz}$. As a result, the bode plot of the intended PLL without RO (i.e. SSLKF-PLL) is shown in Fig.4. It can be seen that the Kalman filter already has some attenuation effect against harmonics considering all potential harmonics are at least $360 \mathrm{~Hz}$.

\section{The choice of $G_{r o}$}

To ensure the stability of RO, poles in (5) must locate within the unity circle. Therefore, the stability criteria for $G_{r o}$ is $0<G_{r o}<2$. $G_{r o}$ is set to 0.5 in this paper.

\section{The choice of $\operatorname{LPF}(z)$}

As mentioned in Section III, it is desired that the (1$R O(z) / z)$ part can be designed to attenuate only the harmonics. When without $\operatorname{LPF}(z)$ (i.e. $\operatorname{LPF}(z)=0$ ), the amplitude response of $(1-R O(z) / z)$ is drawn in Fig.5. As shown, not only the harmonics are attenuated, but also the low frequency region is filtered. Hence, this motivates the use of $\operatorname{LPF}(z)$ to maintain the zero $\mathrm{dB}$ gain in the low frequency region.

In this paper, $\operatorname{LPF}(z)$ is chosen to be a fourth order lowpass filter with cut-off frequency equals $60 \mathrm{~Hz}$ as in (6).

$$
\operatorname{LPF}(z)=\frac{2.381 \times 10^{-8}}{z^{4}-3.95 z^{3}+5.852 z^{2}-3.853 z+0.9512}
$$

It can be seen from Fig. 5 that with $\operatorname{LPF}(z)$, only harmonics within the Nyquist frequency (i.e. $4 \mathrm{kHz}$ ) are attenuated.

\section{Simulation Results}

A simulation model has been built in Matlab/Simulink. The harmonics, unbalance and offset conditions of the intended testing grid are as drawn in Fig.3. According to MILSTD-704 standard, the maximum allowed acceleration for grid frequency variation is $250 \mathrm{~Hz} / \mathrm{s}$ (measured over a period longer than $25 \mathrm{~ms}$ ) under normal operation, and $500 \mathrm{~Hz} / \mathrm{s}$ under abnormal operation. Hence, the grid frequency of the intended three-phase grid is kept $360 \mathrm{~Hz}$ from $0 \mathrm{~s}$ to $1 \mathrm{~s}$. Then, the frequency increases to $800 \mathrm{~Hz}$ following a $500 \mathrm{~Hz} / \mathrm{s}$ slope from $1 \mathrm{~s}$ to $1.88 \mathrm{~s}$ and is kept $800 \mathrm{~Hz}$ for $0.12 \mathrm{~s}$ before decreases to $360 \mathrm{~Hz}$ following a $-250 \mathrm{~Hz} / \mathrm{s}$ slope. Moreover, a 50 -degree phase jump is applied at $0.5 \mathrm{~s}$.

To evaluate the dynamic performance, the proposed DOPLL is compared with SSLKF-PLL at start-up and during a phase jump in subsection $A$ and $B$, respectively. To confirm the steady-state performance, the two PLLs are compared at $360 \mathrm{~Hz}$ in subsection C. Finally, the results during grid frequency variation will be given in subsection D.

\section{A. Performance at start-up}

The original SSLKF-PLL have set the initial values of the observed phase, grid frequency and acceleration all to zero. Since it is already known that the grid frequency will be between $360 \mathrm{~Hz}$ to $800 \mathrm{~Hz}$, the initial value of the observed frequency has been set to $400 \mathrm{~Hz}$ in DO-PLL. Consequently, the transient during start-up is shorter in the proposed DOPLL in Fig.6. The frequency and phase error shown in Fig.6 are the observed frequency $\tilde{f}$ and the error between the real phase $\theta$ and the observed phase $\tilde{\theta}$.
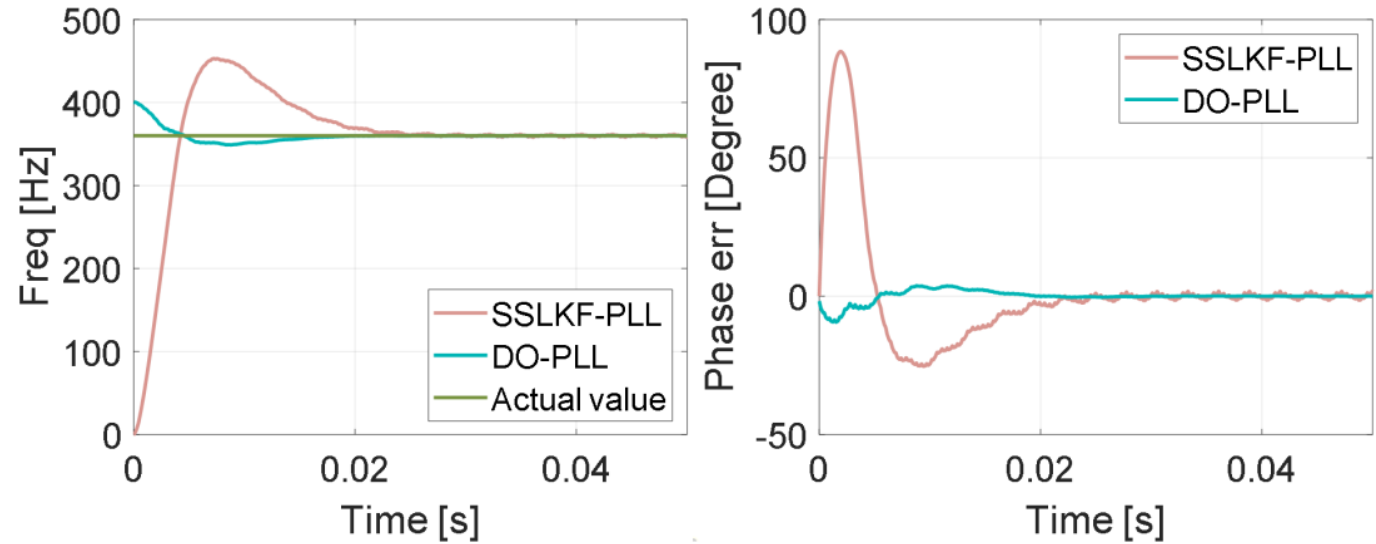

Fig.6: Observed frequency and phase error at start-up.
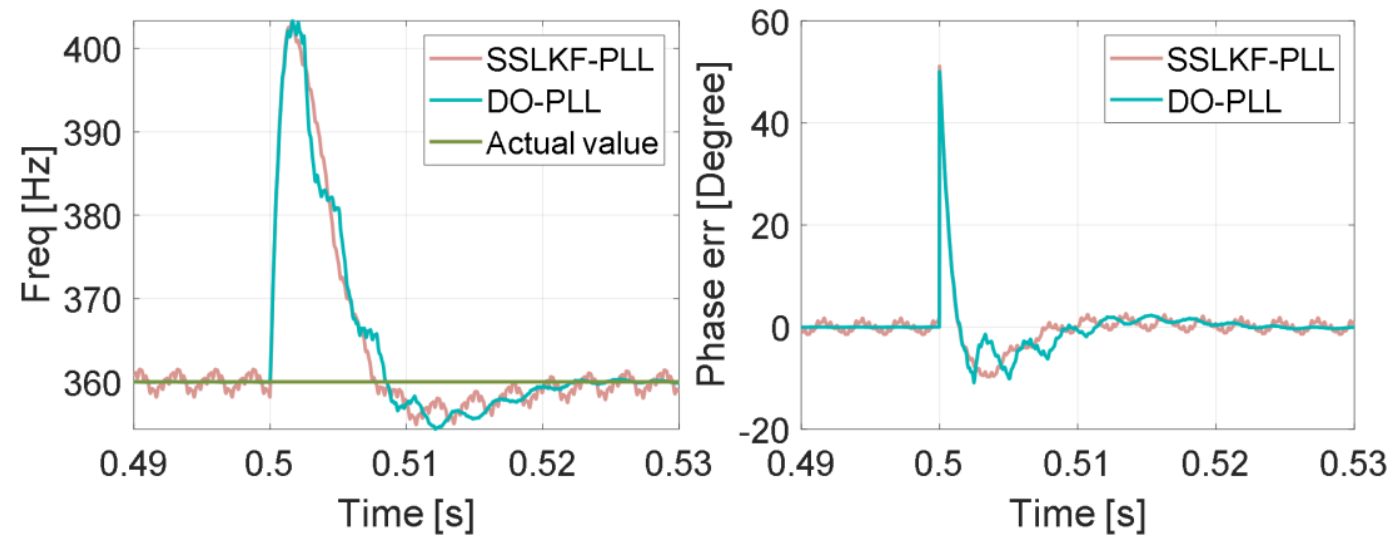

Fig.7: Observed frequency and phase error at phase jump. 


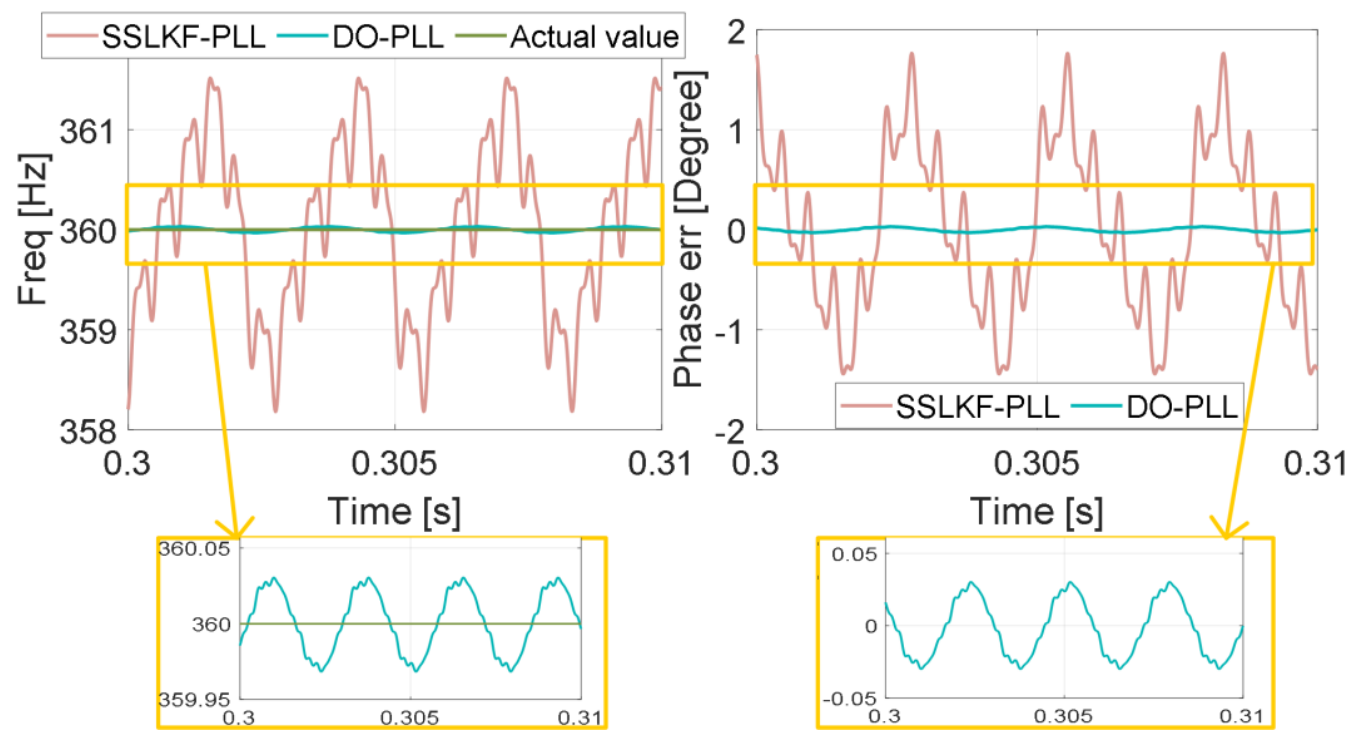

Fig.8: Observed frequency and phase error at $360 \mathrm{~Hz}$.
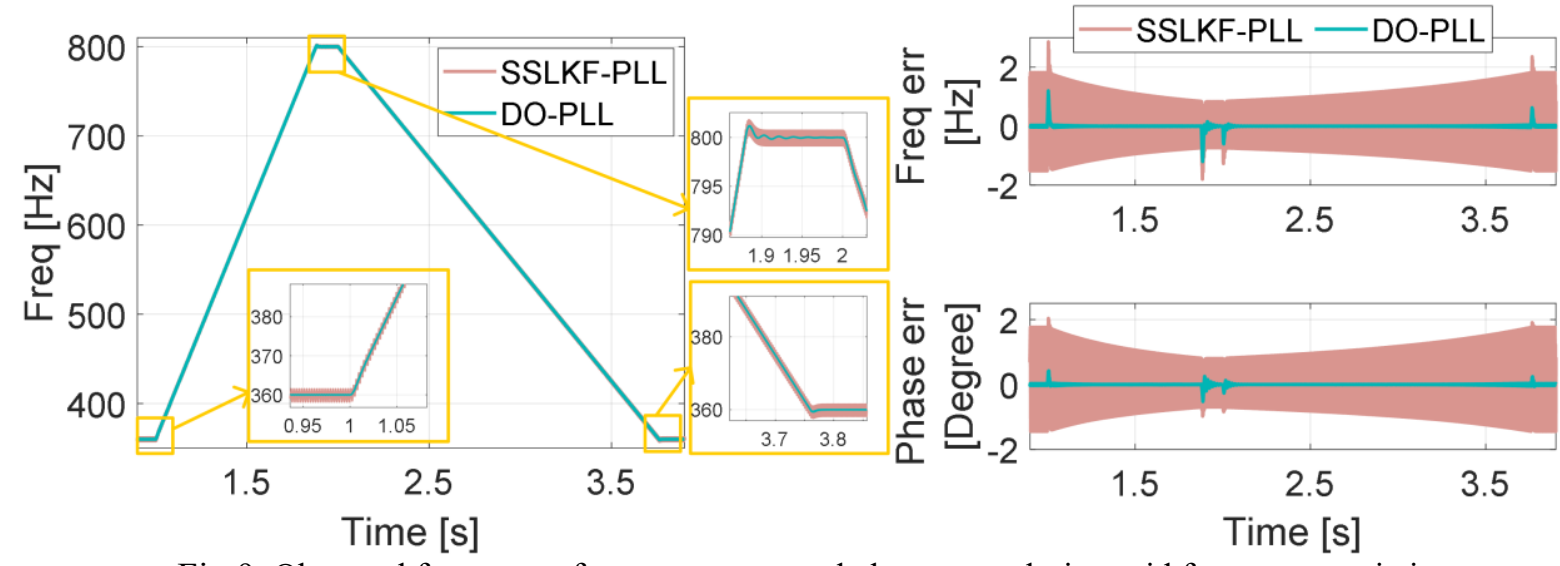

Fig.9: Observed frequency, frequency error and phase error during grid frequency variation.

\section{B. Performance at phase jump}

The observed frequency $\tilde{f}$ and phase error $(\theta-\tilde{\theta})$ during phase jump are shown in Fig.7. It can be seen that the DOPLL and SSLKF-PLL have comparable recover time after the phase jump. This implies that the RO will not affect the transient response while improving the steady-state performance.

\section{Performance at constant grid frequency}

The observed frequency $\tilde{f}$ and phase error $(\theta-\tilde{\theta})$ at $360 \mathrm{~Hz}$ are shown in Fig.8. It can be seen that under $360 \mathrm{~Hz}$ constant grid frequency, DO-PLL reduces the frequency tracking error from $-1.79 \mathrm{~Hz} \sim+1.51 \mathrm{~Hz}$ to $-0.03 \mathrm{~Hz} \sim+0.03 \mathrm{~Hz}$. The phase tracking error is significantly reduced from $-1.42^{\circ} \sim+1.74^{\circ}$ to $-0.03^{\circ} \sim+0.03^{\circ}$.

\section{Performance during grid frequency variation}

The observed frequency $\tilde{f}$, the frequency error $(f-\tilde{f})$ and phase error $(\theta-\tilde{\theta})$ during grid frequency variation are shown in Fig.9. It can be seen clearly from Fig.9 that the DO-PLL has significantly less ripple in its observed frequency $\tilde{f}$ and phase $\tilde{\theta}$.

Another distinctive feature of the $\mathrm{RO}$ is that the disturbance rejection ability of DO-PLL when at $360 \mathrm{~Hz}$ grid frequency is as strong as when at $800 \mathrm{~Hz}$, while the SSLKF-
PPL naturally has increasing tracking error as the grid frequency decreasing.

\section{CONCLUSION}

The repetitive observer (RO) has been added to improve the disturbance rejection ability of the existing steady state linear Kalman filter PLL (SSLKF-PLL) for more electric aircraft (MEA) applications. The SSLKF-PLL is particularly suitable for the variable frequency bus in MEA because it is based on third-order model and can actively compensate the lag/lead in observed frequency and phase due to acceleration/decceleration. By combining RO and SSLKFPLL, a novel dual-observer PLL (DO-PLL) is proposed. Simulation tests considering the worst condition has been carried out. The following benefits of DO-PLL can be concluded:

- Significant reductions of ripple due to harmonics distortions, sensor scaling errors/three-phase unbalances, and d.c. offsets have been achieved.

- RO can suppress the disturbances without affecting the dynamic of the Kalman filter during phase jumps and grid frequency variation.

- Another big convenience of using DO-PLL is that no pre-knowledge of the target disturbance is required. 
It will self-learn and tackle all afore-mentioned disturbances.

- The attenuation of disturbance is always strong regardless of the operating frequency of the grid.

- $\quad$ RO as an add-on tool can be applied to other PLLs operating at different grid frequencies to solve periodic errors.

\section{REFERENCES}

[1] Z. Dai, J. Yang, D. Rao, J. Zhang, and Z. Zhang, "A Global Convergence Estimator of Grid Voltage Parameters for More Electric Aircraft," IEEE Transactions on Industrial Electronics, vol. 67, no. 9, pp. 7540-7549, 2020.

[2] Z. Dai, S. Lv, J. K. Mwaniki, and J. Zhang, "Global accurate estimation of positive- and negative-sequence voltage components for variable frequency AC systems," IET Power Electronics, vol. 11, no. 10, pp. 1730-1737, 2018

[3] H. Yang, Y. Zhang, J. Liang, J. Gao, P. D. Walker, and N. Zhang, "Sliding-Mode Observer Based Voltage-Sensorless Model Predictive Power Control of PWM Rectifier Under Unbalanced Grid Conditions," IEEE Transactions on Industrial Electronics, vol. 65, no. 7, pp. 5550-5560, 2018.

[4] E. Lavopa, P. Zanchetta, M. Sumner, and F. Cupertino, "Real-Time Estimation of Fundamental Frequency and Harmonics for Active Shunt Power Filters in Aircraft Electrical Systems," IEEE Transactions on Industrial Electronics, vol. 56, no. 8, pp. 2875-2884, 2009.

[5] Z. Ž, B. Krstajić, and T. Popović, "Improved Frequency Estimation in Unbalanced Three-Phase Power System Using Coupled Orthogonal
Constant Modulus Algorithm," IEEE Transactions on Power Delivery, vol. 32, no. 4, pp. 1809-1816, 2017.

[6] S. Golestan, J. M. Guerrero, and J. C. Vasquez, "Steady-State Linear Kalman Filter-Based PLLs for Power Applications: A Second Look," IEEE Transactions on Industrial Electronics, vol. 65, no. 12, pp. 9795-9800, 2018.

[7] A. Bellini, S. Bifaretti, and S. Costantini, "A PLL-based filter for speed noise reduction in drives using a resolver transducer," in Proceedings of the 2002 IEEE International Symposium on Industrial Electronics, 2002, pp. 529-534.

[8] A. Bellini and S. Bifaretti, "Performances of a PLL based digital filter for double-conversion UPS," in 2008 13th International Power Electronics and Motion Control Conference, 2008, pp. 490-497: IEEE.

[9] S. Bifaretti, P. Zanchetta, and E. Lavopa, "Comparison of two threephase PLL systems for more electric aircraft converters," IEEE Transactions on Power Electronics, vol. 29, no. 12, pp. 6810-6820, 2014.

[10] M. Tang, A. Formentini, S. A. Odhano, and P. Zanchetta, "Torque Ripple Reduction of PMSMs using a Novel Angle-based Repetitive Observer," IEEE Transactions on Industrial Electronics, 2019.

[11] A. Galassini, G. L. Calzo, A. Formentini, C. Gerada, P. Zanchetta, and A. Costabeber, "uCube: Control platform for power electronics," in 2017 IEEE Workshop on Electrical Machines Design, Control and Diagnosis (WEMDCD), 2017, pp. 216-221.

[12] S. Bifaretti, A. Lidozzi, L. Solero, and F. Crescimbini, "Anti-islanding detector based on a robust PLL," IEEE Transactions on Industry Applications, vol. 51, no. 1, pp. 398-405, 2014.

[13] M. Tang, S. Bifaretti, S. Pipolo, S. Odhano, and P. Zanchetta, "A Novel Repetitive Controller Assisted Phase-Locked Loop with Selflearning Disturbance Rejection Capability for Three-phase Grids," IEEE Journal of Emerging and Selected Topics in Power Electronics, pp. 1-1, 2019. 